\title{
Challenging the Fringe
}

\author{
Jeffrey P. Okeson
}

Published online: 28 December 2013

(C) Indian Prosthodontic Society 2013

Sometimes it is helpful to stand back from the everyday routines of our practices and evaluate the bases for our treatment methods. Our daily routines are often filled with clinical procedures that are successful and proper for our patients. They are proper because we have selected them specifically for our patient's needs. But what makes them so appropriate? Perhaps an analogy may be helpful.

Assume it is 10:00 am on a Monday morning and a 33 year old female reports to your office complaining of difficulty in opening her mouth and pain associated with jaw function. Your first impression is that this patient is suffering from a temporomandibular disorder. Your next instinct would be to reach back into your cortex and retrieve all the information you have stored related to TMD. Your cortex is similar to the hard disc drive on a computer. One file contained on your hard disc drive stores the total body of information that you have been exposed to regarding TMD. It is from this ball of information that you will draw the knowledge necessary to manage your patient's problem. We must begin, however, with one basic fact. The body of information stored in your cortex is incomplete. The reason for this incompleteness may not lie with your inability to acquire the information, but instead with the entire profession's inability to acquire total knowledge in this field. Presently we do not have all the information necessary to manage all of our patients' orofacial pain problems.

Given this reality it is equally important to realize that the body of knowledge you have stored in your cortex is

\section{J. P. Okeson $(\bowtie)$}

Orofacial Pain Program, Department of Oral Health Science, College of Dentistry, University of Kentucky, Lexington, KY 40536-0084, USA

e-mail: okeson@uky.edu not all basic truths. Truth represents fundamental facts or knowledge that cannot be changed or manipulated. Truths are facts that will remain constant over time and can be experimentally duplicated. An example of basic truth or fundamental knowledge is the law of gravity. Once adequately described, this basic knowledge remains unchanged over time, affecting all matter in a very predictable manner. We must accept it as truth.

The ball of information you have stored in your cortex is composed of three different areas or layers. In the center is a hard core area of information composed of basic truths. An example of hard core information in the field of TMD is anatomy. Few of us would argue that the temporalis muscle is a large fan shaped muscle attached to the lateral aspect of the skull reaching inferiorly with a tendinous attachment to the coronoid process of the mandible. When the temporalis muscle shortens, the mandible is elevated. Although there are some minor variations to this anatomy, few of us would debate this fact. It is interesting to note however, that even in the area of anatomy the profession still has debates. The hard core center of your ball of information is made up of basic truths.

Surrounding this hard core information is another layer of information that might be thought of as soft core. The soft core is made up of information that contains some truths, however, we have yet to completely obtained or interpret the data. We acquire soft core information through research documentation. For example, research studies have demonstrated that occlusal appliances significantly reduce symptoms associated with temporomandibular disorders. We presume therefore that this relationship must contain some element of truth. In our attempt to explain this relationship we assumed that the occlusal appliance reduced TMD symptoms by altering the patient's occlusal condition. Following this thought process, it was 
natural to assume that when an occlusal appliance effectively reduced TMD symptoms, the patient's occlusal condition was in some way inadequate and needed to be corrected. The fallacy of this concept lies with the assumption that altering the patient's occlusion is the only method by which the occlusal appliance can alter TMD symptoms. We have since learned that this was a very naive assumption. Occlusal appliances can alter TMD symptoms in many ways, one of which is by altering the patient's occlusal condition. This example demonstrates how truths found in soft core information must be uncovered by interpretation of the data and this interpretation may not always be correct.

The ball of information, made up of its hard core truth center and surrounded by the soft core data is encompassed by a third layer of information called the fringe. The fringe represents information that comes to us totally undocumented by scientific evidence. There is certainly a lot of "fringe" in the area of temporomandibular disorders. The existence of fringe information is not wrong for it contains the ideas that stimulate the learning process. However, the acceptance of this information as if it were hard core truths is often not in the best interest of our patients.

The process of learning is the method by which we uncover additional hard core truths. If we are truly interested in our patient's welfare, our goal should be to increase the hard core truths so that we can more appropriately understand and manage our patient's problems. The manner by which this is achieved is through the scientific method. This process should begin with exploring the soft core information in an attempt to better understand the truth. Let us go back to the occlusal appliance as an example. Although we know that occlusal appliances help reduce TMD symptoms, further investigation may help us learn of the precise relationship between the appliance and alteration of symptoms. For example, assume that 1,000 patients with a specific temporomandibular disorder are selected for a study. Each of these patients are fabricated a specific type of appliance and then, at a set time, their symptoms are re-evaluated by a standard method. Assume that the post treatment data reveals that 250 patients are completely without symptoms, while 250 others reveal no change in symptoms. The remaining 500 patients show partial symptom reduction. If we wish to learn more of how the occlusal appliance affects symptoms we need to take the group of 250 patients who were completely without symptoms and compare them with the group of 250 patients who showed no response at all. By carefully evaluating these subgroups we may identify certain characteristics that differentiate them. Once these characteristics are identified it would be necessary to further investigate them to learn how they may relate to the success or failure of occlusal appliance therapy. Studies such as these help uncover the truths regarding occlusal appliances and TM disorder symptoms. As this information is revealed, the hard core information expands at the expense of the soft core. This is how the orderly body of knowledge increases. It is the process of evidence based dentistry.

Let us once again turn our attention to the fringe. The existence of fringe information is not wrong but in fact very appropriate. As previously mentioned the presence of fringe information stimulates the learning process. Our obligation is to take fringe information and challenge it with scientific studies that will help uncover the truths. If the fringe information has truth within it, scientific investigation will bear this out and the fringe information will move into the soft core area of the body of knowledge. Once it is in the soft core area it is our duty to further investigate it to more precisely reveal the truth. If, on the other hand, the fringe information is investigated and found to be without truth, it should then be discarded and removed from the body of information so as not to confuse us any further.

In my 40 years in the area of temporomandibular disorders, I have seen ideas come and then go, providing no great impact in the area of TMD. Perhaps these ideas contained no basic truths. On the other hand, I have seen some ideas that originally appeared to be without any scientific merit, yet once investigated were documented to contain elements of truth. Many of these ideas have become widely used and are becoming the cornerstones of our treatment.

Now let us go back to the 33 year old female who is in your office seeking care for her temporomandibular disorder. If this were your spouse, mother, father, or child how would you want to determine treatment? I personally would want to be treated with hard core information first. If in fact hard core information did not provide adequate effectiveness, then I would want the therapist to next reach for soft core information. If the soft core information failed to adequately resolve the symptoms, I would then, and only then, consider reaching for the fringe.

As I see it, one of the problems we face today is that many of us are working predominantly with fringe information and do not even realize that it is fringe information. Often this information is presented to us as if it were well documented hard core truths. In past years, this type of therapeutic approach has brought negative publicity to the profession. The use of fringe information can easily result in two types of patient abuse; physical abuse, by changing dental structures when there is no scientific indication for such alterations or, financial abuse, by charging fees that are far exuberant for the time and knowledge provided to the patient. In the past I believe some have been guilty of these abuses.

It appears to me that in the recent years there has been significant improvements by the profession. We are now 
demanding of ourselves far more scientific documentation before treatments are sanctioned. It is to this end that I believe our patients are receiving a far better quality of care for temporomandibular disorders. I commend the changes that have taken place and hope they will continue in the future. I believe it is the obligation of every clinician to question new information for its scientific merit. Separating hard core truths from soft core and fringe information is basic to sound patient management.

Most clinicians appreciate the value of their clinical judgment and frequently use it to determine appropriate treatment. Few of us would care to acknowledge that clinical judgment is often fringe information. Frequently we evaluate a patient, gain a clinical impression and then determine treatment based on our prior successes. Clinical judgment is a routine and important part of the clinical practice. It is not necessarily wrong to base therapy on clinical judgment, however, we should acknowledge that our clinical judgment may not be based on scientific facts. Instead our clinical judgment is more often based on a very small numbers of patients acquired from a skewed population. Therefore when clinical judgment is challenged by scientific evidence, the clinician should be mature and wise enough to depart from clinical judgment so as to be more scientifically sound. This departure from fringe to evidenced based information is another example of the process of learning. Our patients deserve the highest quality treatment that we can offer and this is derived from scientific knowledge. We must continue to challenge our treatment concepts with sound scientific principles so as to uncover more and more truths, enabling better and more effective treatments for our patients. We owe this to our patients and to ourselves. We therefore must constantly challenge the fringe. 